\title{
Membranous glomerulonephritis- A case report
}

\author{
S. Laudari \\ Junior resident, Department of Medicine, College of Medical Sciences, Bharatpur, Nepal
}

\begin{abstract}
Membranous glomerulonephritis is a major cause of nephrotic syndrome and chronic renal insufficiency in adults with a male preponderance. This case has been reported as it is rarely encountered in our clinical practice and its natural course of progression is unpredictable despite immunosuppressive therapies. Male gender, older age, hypertension and persistent proteinuria are associated with worse prognosis.
\end{abstract}

Keywords: Membranous glomerulonephritis, therapies, prognosis.

\section{Introduction}

Membranous glomerulonephritis (MGN) accounts for approximately $30 \%$ of cases of nephrotic syndrome in adults, with a peak incidence between the ages of $30-50$ years and a male to female ratio of $2: 1{ }^{1}$ It may be idiopathic in the majority or secondary to malignancy (solid tumors of the breast, lung, colon), infection (hepatitis B, malaria, schistosomiasis), or rheumatologic disorders like lupus or rarely rheumatoid arthritis in $25-30 \%$ of cases. ${ }^{1,2}$

The condition is characterized by an accumulation of immune deposits on the outer aspect of the glomerular basement membrane, but the target antigens have not been identified. Major contributions to our current understanding of the disease come from Heymann's nephritis, a rat model of membranous glomerulonephritis. ${ }^{3}$

Eighty percent of patients with MGN present with nephrotic syndrome and nonselective proteinuria. ${ }^{1}$ Microscopic hematuria is seen in up to $50 \%$ of patients. ${ }^{1}$ Spontaneous remissions occur in 20-33\%

Correspondences: Dr. S. Laudari

E-mail:Lshankar2@hotmail.com of patients and often occur late in the course after years of nephrotic syndrome. ${ }^{1}$ One-third of patients continue to have relapsing nephrotic syndrome but maintain normal renal function, and approximately another third of patients develop renal failure or die from the complications of nephrotic syndrome. ${ }^{1} \mathrm{MGN}$ has the highest reported incidences of renal vein thrombosis, pulmonary embolism and deep vein thrombosis. ${ }^{1}$

\section{Case report}

A 22 year old male (I.P.No 9694) was admitted in CMS-T.H. on $10^{\text {th }}$ Nov,09 with chief complaint of generalized swelling of the body for 6 months. He first noticed it during his stay in Malaysia for 1 year where he was investigated and sent back to Nepal for further treatment. He was evaluated in detail along with renal biopsy in Kathmandu and started on steroids and pulse methylprednisolone therapy. During the course of treatment, he had few episodes of exacerbation of shortness of breath with hemoptysis which was attributed to left ventricular failure. Investigations of sputum for AFB on repeated samples were negative 64 
and chest X-ray wasn't conclusive either. During his recent admission in hospital, he developed unilateral, tender swelling of the left lower limb which was proven to be Deep Vein Thrombosis on Colour Doppler involving left common iliac, external iliac, common femoral, superficial femoral and popliteal vein including proximal segment of great saphenous vein. He was treated with low molecular weight heparin and warfarin. During the course of treatment, his left leg swelling gradually subsided. One month back, he developed acute right sided chest pain with shortness of breath which was clinically thought to be due to pulmonary embolism. He was managed with anticoagulants and improved. He is on regular follow-up with warfarin, low dose of prednisolone and ACE inhibitor. He has completed 6 cycles of pulse cyclophosphamide therapy. On assessment, his vitals are stable. He has cushingoid face and acneiform facial eruptions (secondary to steroids) with $\mathrm{BMI}=18$. He has mild pedal edema and ascites without features of heart failure and is passing adequate urine. Before initiating therapy, he had deranged renal function tests, dyslipidemia (raised LDL,TGs and cholesterol), hematuria and proteinuria $(++++),(6 \mathrm{gms}$ in 24 hours urine) along with granular casts in urine R/E. ANA was negative. USG abdomen showed normal size kidneys with loss of corticomedullary differentiation suggestive of medical renal disease-Gr II and ascites.

\section{Kidney biopsy on microscopic evaluation reveals:}

\begin{tabular}{llll}
\hline Glomeruli & Tubules & Interstitium & Blood vessels \\
\hline Total: 9 & Casts absent & Inflammation absent & Thickening absent \\
Cellularity: normal, One glomerulus partially & & \\
sclerosed, absence of crescents, & & \\
cellular proliferation, deposits, & & \\
polymorphs and mild basement & & \\
membrane thickening & & \\
\hline
\end{tabular}

On immunofluorescence assay, IgG(positive, peripheral, coarse granular), IgA(no glomerular deposits seen), $\operatorname{IgM}$ ( no glomerular deposits seen) and C-3(positive, peripheral, coarse granular). IgA and IgM shows autofluorescence in tubular casts. C3 shows autofluorescence in blood vessels. Negative control slide does not show any fluorescence. Final diagnosis was suggestive of Membranous Glomerulonephritis.

Now, patient is on regular follow-up. He still has mild bilateral pedal edema and ascites. He does not have further episodes of hemoptysis and shortness of breath. He has proteinuria ( $2.5 \mathrm{gms}$ in 24 hours urine) without hematuria and dyslipidemia. His renal parameters, random blood sugar, total serum protein and rest of the routine investigations are within normal range.

\section{Discussion}

Three possible mechanisms have been suggested for MGN:2

1. Glomerular localization of preformed,circulating immune complexes composed of low molecular weight oligovalent antigen and low affinity IgG antibody. 
2. Reaction of circulating antibody with an intrinsic glomerular antigen discontinuously distributed along lamina rara externa resulting in formation of immune complexes in situ.

3. Reaction of circulating antibody with an antigen normally extrinsic to the glomerulus but planted there by virtue of some biochemical or electrostatic affinity for GBM.

The immune complex serves as an activator that triggers a response from the $\mathrm{C} 5 \mathrm{~b}-\mathrm{C} 9$ complements, which form a membrane attack complex (MAC) on the glomerular epithelial cells. ${ }^{2}$

While different stages (I-V) of progressive membranous lesions have been described, some published analysis indicate that the degree of tubular atrophy or interstitial fibrosis is more predictive of progression than the stage of glomerular disease. ${ }^{2}$

Clinically, the majority will have heavy proteinuria and other features of nephrotic syndrome and the remainder will present with symptomless proteinuria with or without hematuria. Onset is usually insidious and without antecedent upper respiratory infection. Hypertension and azotemia occur late in the course of the disease at the time of onset of nephrotic syndrome but in 1/3-3/4 of cases, it is occult at the time of discovery of the nephrotic syndrome. ${ }^{2}$

On laboratory evaluation, proteinuria is over $3.5 \mathrm{~g} /$ day in over $80 \%$ of cases. ${ }^{2}$ It is nonselective but highly selective proteinuria may occur in as many as $20 \%$ of cases. ${ }^{2,4}$ Increased excretion of membrane attack complex neoantigen may be indicative of MGN in an active stage. Microscopic hematuria is common whereas gross hematuria is rare. Levels of $\mathrm{C} 3$ and other complement components are nearly always normal.

\section{Course and therapy}

Course of idiopathic MGN is indolent and slowly progressive, at times punctuated by clinical remissions and exacerbations of the nephrotic syndrome. The overall prognosis in children is excellent but the frequency of spontaneous complete remission of proteinuria in adults varies among series but averages about 25 percent. ${ }^{5}$ An additional 20-25 percent of cases sustain a spontaneous partial remission (i.e. protein excretion less than 2gm but more than $200 \mathrm{mg} /$ day) and maintain a stable GFR for extended period. ${ }^{6}$

An extensive retrospective survey conducted by the Mayo clinic Group demonstrated a 10 year patient survival of about 75 percent regardless of whether patients were treated with steroids or immunosuppressive drugs or remained untreated; 20 percent developed end stage renal failure of which 60 percent occurred within 2.5 years of diagnosis (so called rapidly progressive MGN). ${ }^{7}$ Similar findings have been reported from France, Japan, Finland and Scotland. ${ }^{7}$ Male sex, older age at onset, severe proteinuria (>10gm/day), poorly controlled HTN, severe hypercholesterolemia, reduced GFR at onset and renal biopsy findings of advanced glomerular disease (stage III or IV), tubule atrophy and interstitial fibrosis have all been associated with poor prognosis and a high risk for subsequent development of progressive renal failure. ${ }^{8}$

Hopper and co-workers and Bolton and colleagues in uncontrolled studies have suggested that higher dosage and/or more prolonged therapy (i.e. 6 months to several years) with daily or alternate-day steroids is associated with an improved outcome, however it has more side effects. ${ }^{2}$

The precise role of cytotoxic therapy is uncertain but its possible role in MGN has been suggested by 
several controlled and uncontrolled studies. Addition of cyclophosphamide to alternate day or daily steroids has been associated with reversal of progressive renal insufficiency or decrease in proteinuria. Ponticelli and colleagues have shown in a controlled study that sequential therapy with high dose intravenous methylprednisolone, oral methylprednisolone and chlorambucil over 6 months can result in increased likelihood of complete or partial remission and better maintenance of renal function. ${ }^{9}$ Other therapies include tacrolimus and mycophenolate mofetil.

\section{Conclusion}

Membranous glomerulonephritis has risk of coagulopathy including deep vein thrombosis and renal vein thrombosis. It has unpredictable course and outcome. The therapies include steroids and immunosuppressive agents.

\section{References}

1. J.B.Lewis, Eric G Neilson. Glomerular diseases. Dennis L Kasper, Anthony Fauci, Ran Longo et al. Harrison's principles of internal medicine. $17^{\text {th }}$ ed. Newyork: Mc Graw-Hill; 2008; 277: 1789-90.

2. R.J.Glassock, S.G. Adler, H.J.Ward, et al. Primary glomerular diseases. Brenner, BM and Rector FC Jr. The Kidney, $3^{\text {rd }}$ ed. Philadelphia: WB Saunders; 1986: 929.
3. W. Heymann, D.B.Hackel, S. Harwood et al. Production of nephrotic syndrome in rats by Freund's adjuvants and rat kidney suspension. Proc Soc Exp Biol Med 1959; 100: $660-4$.

4. C.H. Coggins, J.P. Frommer, R.J. Glassock. Mebranous nephropathy. Sem. Nephrol. 1982; 2: 264.

5. J. Ehrenreich, J.Churg. Pathology of membranous nephropathy. Pathol. Ann. 1968; 3: 145.

6. P. Zuccheli, C. Cagnoli, S. Pasquali, et al. Clinical and morphologic evolution of idiopathic membranous nephropathy. Clin. Nephrol. 1986; 25: 282.

7. H. Kida, T. Asamata, H. Yokoyoma, et al. Long term prognosis of membranous glomerulonephritis. Clin. Nephrol. 1986, 25: 64.

8. W.H. Tu, D.B. Petitti, C.G. Biara, et al. Membranous nephropathy: Predictors of terminal renal failure. Nephron. 1984; 36: 118.

9. C. Ponticelli, P. Zuchheli, P. Possevini, et al. A randomized trial of methylprednisolone and chlorambucil in idiopathic membranous nephropathy. N. Eng. J.Med. 1989; 320: 8. 